Anaesthesist 2012 $\cdot 61: 674-675$

DOI 10.1007/s00101-012-2056-7

Online publiziert: 28. August 2012

(c) Springer-Verlag 2012

\section{Blobner}

Klinik für Anästhesiologie, Technische Universität München, Klinikum rechts der Isar, München

\title{
Wenn Kasuistiken fehlende Evidenz ersetzen müssen
}

In Zeiten der evidenzbasierten Medizin mutet es ungewöhnlich an, wenn eine Kasuistik in einer wissenschaftlichen Zeitschrift wie Der Anaesthesist von einem Editorial begleitet wird. Wichtige Fragestellungen der Anästhesiologie werden in kontrollierten Studien mit ausreichenden Fallzahlen untersucht. Fallberichte sind meistens für seltene Verläufe mit besonderen Merkmalen vorbehalten, die gelegentlich auch nicht verallgemeinert werden können. Was ist also an der Fallserie von Nauheimer et al. zur modifizierten Blitzintubation bei 10 Kaiserschnitten so besonders, dass es eines weiteren Kommentars bedarf?

Es gibt jährlich unzählige Anästhesien für Kaiserschnitte in Deutschland und weltweit. Trotz des Vormarsches der Regionalanästhesieverfahren werden auch noch immer ausreichend viele Kaiserschnitte in Allgemeinanästhesie durchgeführt, um systematische Untersuchungen im Grundsatz zu ermöglichen. Auch ist glücklicherweise bei keiner der beschriebenen 10 Patientinnen und ihrer Kinder ein besonderer oder ungewöhnlicher Verlauf beobachtet worden, der berichtenswert wäre. Und trotzdem ist es bereits die zweite Fallserie zum Einsatz von Rocuronium zur „rapid sequence induction“ (RSI) für eine Allgemeinanästhesie zum Kaiserschnitt nach dem kürzlich erschienenen sehr ähnlichen Beitrag von Pühringer et al. [11], die genauso wie Nauheimer et al. mögliche Restblockaden am Ende der Operation bedarfsadaptiert mit Sugammadex reversiert haben.
Die einzelnen „Komponenten“ für das Vorgehen in den beiden Fallserien sind ausreichend belegt:

1. Die schnelle Reversierung von möglichen Restblockaden nach Rocuronium am Operationsende mit Sugammadex ist hinlänglich bekannt $[4,8]$.

2. Rocuronium in einer Dosis von 1,0$1,2 \mathrm{mg} / \mathrm{kgKG}$ erlaubt vergleichbar gut und schnell zu intubieren wie Succinylcholin [10].

Welche Konsequenzen haben diese Fakten auf die anästhesiologische Praxis? Zwar verwenden immer mehr Anästhesisten Rocuronium für die RSI, trotzdem entscheiden sich noch immer die meisten für Succinylcholin [5]. Als wichtigstes Argument für Succinylcholin gilt noch immer dessen kurze Wirkungszeit. Dabei ist in aller Regel der Gedanke führend, dass im Fall der in der Geburtshilfe häufiger auftretenden Schwierigkeiten mit dem Atemwegsmanagement eine rechtzeitig einsetzende Spontanatmung Mutter und Kind vor Hypoxie schützen könnte. Dass es sich dabei v. a. um eine gefühlte Sicherheit handelt, ist wohl inzwischen jedem Anästhesisten - also auch den Succinylcholinanwendern - bewusst. Gerade in der Kaiserschnittsituation sind die Sauerstoffreserven kaum ausreichend, bis die neuromuskuläre Erholung nach Succinylcholin eine rettende Spontanatmung wieder erlauben würde. Daher haben die verschiedensten Atemwegshilfen, eingebettet in Algorithmen, längst einen viel wichtigeren Stellenwert zur Vermeidung dieses anästhesiologischen Horrorszenarios eingenommen [1].
Angesichts dieser gesicherten Datenlage erhoffen sich vermutlich die meisten Anästhesisten bereits beim Lesen der Überschrift neuere Erkenntnisse zur Situation des "Can't intubate - can't ventilate“ beim Kaiserschnitt und zu einem möglichen Nutzen von Sugammadex in einer solchen Situation. Diese Hoffnung wird schließlich von der Erkenntnis genährt, dass die Kombination aus 1,0-1,2 mg/ $\mathrm{kgKG}$ Rocuronium und 16 mg/kgKG Sugammadex eine schnellere neuromuskuläre Erholung ermöglicht als die Spontanerholung nach $1,0 \mathrm{mg} / \mathrm{kgKG}$ Succinylcholin [9]. Glücklicherweise können beide Fallserien nicht von einem solchen Sugammadexeinsatz im Rahmen eines Atemwegsproblems berichten. Während Pühringer et al. auf diese Frage auch nicht explizit eingehen, diskutieren Nauheimer et al. die Optionen durch Sugammadex in diesem Zusammenhang.

Die von der European Medicines Agency veröffentlichte Produktinformationen erlaubt ausdrücklich sowohl bei schwangeren als auch bei stillenden Frauen Sugammadex zu verwenden [2]. Damit wenden die Anästhesisten in beiden Fallserien - zumindest auf den ersten Blick Sugammadex auch im Rahmen der Zulassung an. Schwieriger wird die Frage jedoch, wenn Sugammadex vor der Entwicklung des Kindes in einer Dosis von $16 \mathrm{mg} / \mathrm{kgKG}$ injiziert würde. Das Neugeborene würde damit Sugammadex erhalten, dessen Einsatz jedoch erst ab dem zweiten Lebensjahr zugelassen ist. Vermutlich ist diese „indirekte“ Gabe von Sugammadex eher eine juristisch zu klärende Frage. Am einfachsten wäre trotz- 
dem ein konkreter Hinweis des Herstellers zur Anwendung von Sugammadex im beschriebenen Szenario, denn bislang macht die Produktinformation Aussagen weder zur Zulassung noch zur „Nichtzulassung" von Sugammadex beim Kaiserschnitt.

\section{Dünnes juristisches Eis}

Nauheimer et al. begründen die Gabe von Sugammadex vor der Entwicklung des Kindes mit „... der Abwendung weiterer schwerwiegender Komplikationen ..." Dem möchte ich als Arzt nur vollstens zustimmen. Trotzdem befallen mich Zweifel, wie dieses Vorgehen im Fall eines Haftpflichtschadens bewertet würde. Denn, um im Notfall auch wirklich schnell genug $16 \mathrm{mg} / \mathrm{kgKG}$ injizieren $\mathrm{zu}$ können, muss Sugammadex vor der Narkoseeinleitung vorbereitet sein [3]. Damit ist Sugammadex Teil des Anästhesiekonzepts, das den Einsatz schon vor der Entwicklung des Kindes impliziert. Über einen solchen Plan muss dann aber auch individuell aufgeklärt werden, falls es sich - wie befürchtet - um einen nichtzugelassenen Einsatz („off label use“) von Sugammadex handeln sollte. Wie schwierig oder gar unmöglich so detaillierte Aufklärungsinhalte gerade in der geburtshilflichen Anästhesie sind, ist hinlänglich bekannt [6].

Liegt darin letztlich der Wert von Fallserien für die geburtshilfliche Anästhesie? Kann das Kalkül aufgehen, dass mithilfe einer ausreichend hohen Zahl von berichteten Fällen quasi der „Gewohnheitsgebrauch" eines neuen Medikaments für die schwierige Zulassung von geburtshilflichen Indikationen unterstützt wird? Oder droht eher das umgekehrte Szenario, dass eine Erwartungshaltung, diese Medikamente einzusetzen, entsteht, obwohl die Zulassung nicht geklärt ist? Die Tatsache, dass wir für die Allgemeinanästhesie zum Kaiserschnitt noch immer die 1959 beschriebene Technik verwenden [7], lässt mich an beiden Vermutungen zweifeln. Fallberichte können uns lehren, wie wir vielleicht bessere Behandlungskonzepte entwickeln können. Am Ende müssen dann jedoch kontrollierte Studien an ausreichend großen Fallzahlen den Nutzen auch dieser Technik belegen.
Das gilt auch oder gerade, weil es sich um eine emotional schwierige Fragestellung in der geburtshilflichen Anästhesiologie handelt. Die Hemmnisse für solche Studien sind gewaltig. Ethikkommissionen und Versicherungsbedingungen sind gerade bei Studien in der geburtshilflichen Anästhesie besonders kritisch, genauso wie die zukünftigen Eltern und die Geburtshelfer. Das ist sicherlich notwendig und wichtig, sollte jedoch nicht dazu führen, dass ebenso notwendige und wichtige klinische Studien nicht durchgeführt werden können.

Nauheimers sorgfältig aufgearbeitete Fallserie zeigt uns den Weg zu einer sehr wichtigen klinischen Untersuchung in der geburtshilflichen Anästhesie.

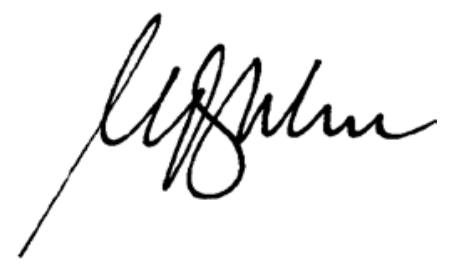

M. Blobner

\section{Korrespondenzadresse}

\section{Prof. Dr. M. Blobner}

Klinik für Anästhesiologie,

Technische Universität München,

Klinikum rechts der Isar

Ismaninger Str. 22, 81675 München

blobner@lrz.tu-muenchen.de

\section{Literatur}

1. American Society of Anesthesiologists Task Force on Management of the Difficult Airway (2003). Practice guidelines for management of difficult airway. Anesthesiology 98:1269-1277

2. European Medicines Agency (2008) http://www. ema.europa.eu/docs/de_DE/document_library/ EPAR_-_Product_Information/human/000885/ WC500052310.pdf

3. Bisschops M, Holleman C, Huitink J (2010) Can sugammadex save a patient in a simulated,cannot intubate, cannot ventilate' situation? Anaesthesia 65:936-941

4. Blobner M, Eriksson LI, Scholz J et al. (2010) Reversal of rocuronium-induced neuromuscular blockade with sugammadex compared with neostigmine during sevoflurane anaesthesia: results of a randomised, controlled trial. Eur J Anaesthesiol 27:874-881

5. Fink H, Geldner G, Fuchs-Buder T et al. (2006) Muskelrelaxanzien in Deutschland 2005: Ein Vergleich zwischen den Anwendungsgewohnheiten in Krankenhäusern und Praxen. Anaesthesist 55: 668-678
6. Goecke T, Bender H, Lorenz C et al. (2001) Periduralanalgesie unter der Geburt. Aufklärung und Dokumentation aus geburtshilflicher, anästhesiologischer und juristischer Sicht. Gynäkologe 34:458-462

7. Hodges R, Bennett J, Tunstall M et al. (1959) General anaesthesia for operative obstetrics: with special reference to the use of thiopentone and suxamethonium. Br J Anaesth 31:152-163

8. Jones RK, Caldwell JE, Brull SJ et al. (2008) Reversal of profound rocuronium-induced blockade with sugammadex: a randomized comparison with neostigmine. Anesthesiology 109:816-824

9. Lee C, deSilva AJ (1979) Acute and subchronic neuromuscular blocking characteristics of streptomycin: a comparison with neomycin. Br J Anaesth 51:431-434

10. Perry JJ, Lee JS, Sillberg VA et al. (2008) Rocuronium versus succinylcholine for rapid sequence induction intubation. Cochrane Database Syst Rev:CD002788

11. Pühringer $F$, Kristen $P$, Rex $C$ (2010) Sugammadex reversal of rocuronium-induced neuromuscular block in Caesarean section patients: a series of seven cases. Br J Anaesth 105:657-660 